\title{
Über die Verallgemeinerung eines Gauß'schen Algorithmus.
}

Von Dr. Max Mandl.

In den Monatsberichten der Akademie der Wissenschaften zu Berlin (Jahrgang 1876, S. 330) gibt Herr Schering in Göttingen eine Verallgemeinerung des Gau'schen Kriteriums für den quadratischen Restcharakter einer Zahl in Bezug auf eine andere, welche er folgendermaßen ausspricht:

"Handelt es sich $\mathrm{um}$ den Rest $A$ und den Theiler $P$, welcher eine Primzahl oder eine zusammengesetzte Zahl sein kann, aber zu $2 A$ als relativ prim vorauszusetzen ist, und stellen wir die von $\mathrm{G}$ a u $\beta$ betrachtete Eigenschaft nach der von Jacobi so zweckmäßig eingeführten Verallgemeinerung des Lege ndre'schen Zeichens dureh den Wert +1 oder -1 dar, so wird

$$
\left(\frac{A}{P}\right)=(-1)^{\mu},
$$

worin $\mu$ die Anzahl der negativen Reste bedeutet, welche entstehen, wenn man von den Producten

$$
\text { 1. } A, 2 . A, 3 . A, \ldots \frac{P-1}{2} \cdot A
$$

die absolut kleinsten Reste für den Theiler $P$ bildet."

Anschließend hieran (S. 331-341) theilt Herr Kro ne cker den erforderlichen Beweis dieses Satzes und seine auf denselben Gegenstand bezüglichen Untersuchungen mit. Herr Kronecker geht von der Eisenstein'schen Darstellung für das Liegendre'sche Symbol

$$
\left(\frac{r}{n}\right)=\prod_{R}\left(\frac{\sin \frac{2 r K \pi}{n}}{\sin \frac{2 K \pi}{n}}\right)
$$

aus, welche unter der Voraussetzung abgeleitet worden war, dass $n$ eine Primzahl bedeutet, und untersucht den Wert des in Gleichung (1) auf der rechten Seite stehenden Productes für den Fall, dass jene Beschränkung aufgehoben wird. Er findet für dasselbe eine Reihe von Eigenschaften, aus denen sich schließlich ergibt, dass, wenn $n$ eine Primzahl ist, die fragliche 
Größe mit dem Le ge ndre'schen Symbol, anderenfalls aber mit dem Jacobi'schen Symbol $\left(\frac{r}{n}\right)$ identisch ist. Durch arithmetische Interpretation folgt sodann aus Gleichung (1) die Richtigkeit des zu beweisenden Satzes.

Es soll nun im Folgenden ein Beweis des KroneckerSchering'schen Satzes gegeben werden, welcher sich auf eine eigenthümliche Classeneintheilung der Zahlen $1,2,3, \ldots \frac{P-1}{2}$ gründet. Des bequemeren Ausdruckes halber soll hier die oben erwähnte Reihe der absolut kleinsten Reste modulo $P$, gebildet aus sämmtlichen Gliedern der Reihe $(A)$, hingeschrieben werden; von der Reihenfolge der Glieder abgesehen, lautet dieselbe offenbar:

$$
\varepsilon_{1} \cdot 1, \varepsilon_{2} \cdot 2, \varepsilon_{3}, 3 \ldots \varepsilon_{\frac{P-1}{2}} \cdot \frac{P-1}{2}
$$

Die Gründe dafür sind genau dieselben wie in den Fällen, wo $P$ eine Primzahl ist.

Der zu beweisende Satz kann dann auch durch die Gleichung

$$
\left(\frac{A}{P}\right)=\varepsilon_{1} \cdot \varepsilon_{2} \cdot \varepsilon_{3} \cdot \ldots \frac{\varepsilon_{P-1}}{2}
$$

ausgedrückt werden; dem Beweise desselben möge jedoch folgender Hilfssatz vorangeschickt werden:

Ist $P$ eine ungerade $\mathrm{Zahl}$ und $A$ relativ prim zu $P$, so ist

$$
\left.\begin{array}{ll}
\text { entweder } A^{\frac{1}{2} \varphi(P)} & =\left(\frac{A}{a}\right) \\
\text { oder } \quad A^{\frac{1}{2} \varphi(P)} & \equiv 1
\end{array}\right\}(\bmod P),
$$

(unter $\left(\frac{A}{a}\right)$ das Legendre'sche Symbol verstanden), je nachdem $P$ nur den einzigen Primfactor $a$, oder deren mehrere enthält.

Es ist dies in folgender Weise einzusehen: Sei zunächst $P=a^{\alpha}$, so hat man nach Euler

oder

$$
\begin{aligned}
& A^{\frac{a-1}{2}} \equiv\left(\frac{A}{a}\right)(\bmod a) \\
& A^{\frac{a-1}{2}}=\left(\frac{A}{a}\right)+K \cdot a
\end{aligned}
$$

und nach wiederholter Potenzierung mit $u$ :

$$
A^{a^{a-1} \cdot \frac{a-1}{2}}=\left(\frac{A}{a}\right)+K_{1} \cdot a^{\alpha}
$$


oder

$$
\begin{gathered}
A^{a^{\alpha-1} \cdot \frac{a-1}{2}} \equiv\left(\frac{A}{a}\right)\left(\bmod a^{\alpha}\right) \\
a^{\alpha-1} \cdot \frac{a-1}{2}=\frac{1}{2} \varphi(P): \\
A^{\frac{1}{2} \varphi(P)} \equiv\left(\frac{A}{a}\right)(\bmod P),
\end{gathered}
$$

und nachdem

wie behauptet worden war.

Ist dagegen zweitens $P=a^{\alpha} . b^{\beta} . c^{\gamma} \ldots$, worin $a, b, c, \ldots$ verschiedene ungerade Primzahlen bedeuten, so hat man nach dem ersten Falle:

und ebenso: $\quad A^{\frac{1}{2} b^{\beta-1} \cdot(b-1)} \equiv\left(\frac{A}{b}\right)\left(\bmod b^{\beta}\right)$

$$
A^{\frac{1}{2} c^{\gamma-1} \cdot(c-1)} \equiv\left(\frac{A}{c}\right)(\bmod c \gamma)
$$

Werden diese Congruenzen der Reihe nach mit

$$
b^{\beta-1}(b-1) c^{\gamma-1}(c-1) \ldots,
$$

beziehungsweise mit $a^{\alpha-1}(a-1) c^{\gamma-1}(c-1) \ldots$ etc. potenziert, so erhält man auf der linken Seite überall $A^{\frac{1}{2} \varphi(P)}$, auf der rechten Seite aber durchwegs +1 , weil nur mit geraden Zahlen potenziert wurde, und man hat daher:

$$
A^{\frac{1}{2} \varphi(P)}=1
$$

nach jedem der moduli $a^{\alpha}, b^{\beta}, c^{\gamma} \ldots$ also auch nach deren Producte $a^{\alpha} . b^{\beta} . c^{\gamma} \ldots ;$ man hat daher in diesem Falle

$$
A^{\frac{1}{2} \varphi(P)} \equiv 1(\bmod P)
$$

womit der Hilfssatz bewiesen erscheint.

Um nun den Wert des Productes $\varepsilon_{1} \varepsilon_{2} \varepsilon_{3} \ldots \frac{\varepsilon_{P-1}}{2}$ zu ermitteln, theilen wir alle Glieder der Reibe $(A)$ in Gruppen ein, 
und berechnen für die zugehörigen Glieder von $(B)$ das Product aller $\varepsilon$-Größen. In eine und dieselbe Gruppe sollen alle jene Glieder $m A$ von (A) eingereiht werden, deren Coefficienten $m$ einen und demselben größten gemeinschaftlichen Theiler $\delta$ mit $P$ besitzen (und im Intervalle 1 bis $\frac{P-1}{2}$ liegen).

Lässt man sodann $\delta$ der Reihe nach alle möglichen Theiler von $P$ bedeuten, wobei die Einheit mit einzurechnen und nur $P$ selbst anszuschließen ist, weil $F>\frac{P-1}{2}$, so füllen die entsprechenden Gruppen der Zahlen $m$ läckenlos und ohne Wiederholung die Zahlenreihe $1,2,3, \ldots \frac{P-1}{2}$ aus, die entsprechenden Gruppen der Zahlen $m A$ dagegen die Reihe $(A)$.

Suchen wir zunächst alle Glieder von $(A)$, welche überhaupt durch $\delta$ theilbar sind, so bilden diese die Reihe

$$
\delta A, 2 \delta A, 3 \delta A \ldots \frac{1}{2}\left(\frac{P}{\delta}-1\right) \ldots(C)
$$

und diese Reihe muss mit $\frac{1}{2}\left(\frac{P}{\delta}-1\right) . \delta A$ schließen, weil

$$
\frac{1}{2}\left(\frac{P}{\delta}-1\right) \delta A=\frac{P-\delta}{2} \cdot A \leq \frac{P-1}{2} \cdot A,
$$

während schon das nächste Glied der Reihe $(\dot{C})$ :

$$
\frac{1}{2}\left(\frac{P}{\delta}+1\right) \cdot \delta A=\frac{P+\delta}{2} \cdot A>\frac{P-1}{2} \cdot A,
$$

also nicht mehr unter den Gliedern von $(A)$ enthalten wäre.

Damit nun $\delta$ auch der größte gemeinschaftliche Factor zwischen dem Gliede $r \delta A$ der Reihe $(C)$ und der Zahl $P$ sei, ist nothwendig und hinreichend, dass $r$ relativ prim zu $\frac{P}{\delta}$ sei. Die Anzahl aller solcher Glieder $r \delta A$ ist demnach gleich der Anzahl aller Zahlen, welche nicht größer als $\frac{1}{2}\left(\frac{P}{\delta}-1\right)$ und relativ prim zu $\frac{P}{\delta}$ sind. Ist aber $r$ relativ prim $\mathrm{zu} \frac{P}{\delta}$, so ist $\frac{P}{\delta}-r$ von derselben Beschaffenheit, und da $\frac{P}{\delta}$ ungerade ist, so zerfallen die $\varphi\left(\frac{P}{\delta}\right)$ Zahlen, welche $z \mathfrak{u} \frac{P}{\delta}$ relativ prim und kleiner als $\frac{P}{\delta}$ sind, genau in zwei Hälften, von denen die erste im Intervalle 1 bis $\frac{1}{2}\left(\frac{P}{\delta}-1\right)$ liegt, die zweite aber außerhalb dieses Intervalls. Die Anzahl aller Zahlen $r$ von der fraglichen Beschaffenheit ist demnach gleich $\frac{1}{2} \varphi\left(\frac{P}{\delta}\right)$. 
Betrachten wir nun irgend ein Glied $r \delta A$ und reducieren wir dasselbe auf seinen absolut kleinsten Rest modulo $P$, so muss dieser nothwendig auch durch $\delta$ theilbar sein, also die Form $\varepsilon s \delta$ haben, worin $\varepsilon= \pm 1$ das Vorzeichen des Restes bedeutet. Es muss aber auch $\delta$ der g rö $b$ te gemeinschaftliche Theiler dieses Restes und der Zahl $P$ sein, d. h. die Zahl $s$ ist relativ prim zu $\frac{P}{\delta}$, und da sie überdies im Intervalle 1 bis $\frac{1}{2}\left(\frac{P}{\delta}-1\right)$ enthalten sein muss, so ist $s$ genau von derselben.Beschaffenheit wie $r$.

Es besteht also zunächst die Congruenz:

$$
\begin{aligned}
r \delta A & \equiv \varepsilon \delta s(\bmod P) \\
\text { oder } r A & \equiv \varepsilon s\left(\bmod \frac{P}{\delta}\right)
\end{aligned}
$$

und wenn man $r$ alle seine $\frac{1}{2} \varphi\left(\frac{P}{\delta}\right)$ Werte durchlaufen lässt und die erhaltenen Congruenzen mit einander multipliciert, so erhält man:

$$
\prod(\cdot) \cdot A^{\frac{1}{2} \varphi\left(\frac{P}{\delta}\right)} \equiv \prod(\varepsilon) \cdot \prod(s)\left(\bmod \frac{P}{\delta}\right)
$$

Es ist klar, dass zwei verschiedenen Werten von $r$ auch ungleiche Werte ron $s$ entsprechen, denn sonst könnten die beiden folgenden Congruenzen neben einander bestehen:

$$
\left.\begin{array}{l}
r A \equiv \varepsilon^{\prime} s \\
r^{\prime} A \equiv \varepsilon^{\prime} s
\end{array}\right\}\left(\bmod \frac{P}{\delta}\right)
$$

( $\varepsilon^{\prime}$ ebenfalls gleich \pm 1 ), woraus mit Rücksicht auf die Be.deutung von $\varepsilon$ und $\varepsilon^{\prime}$ folgen würde, dass

$$
\left.\begin{array}{ll}
\text { entweder } & \left(r+r^{\prime}\right) A=0 \\
\text { oder } & \left(r-r^{\prime}\right) A \equiv 0
\end{array}\right\}\left(\bmod \frac{p}{\delta}\right),
$$

was unmöglich ist, nachdem $A$ relativ prim zu $\frac{P}{\delta}$, ferner $r$ und $r^{\prime}$ nach der Voraussetzung von einander verschieden und einzeln höchstens gleich sind $\frac{1}{2}\left(\frac{P}{\delta}-1\right)$.

Hieraus folgt, dass, wenn $r$ alle zulässigen Werte durchläuft, $s$ in geänderter Reihenfolge dieselben $\frac{1}{2} \varphi\left(\frac{P}{d}\right)$ Werte annimmt, so dass in Congruenz (1)

$$
\Pi(r)=\Pi(s)
$$

and da jedes einzelne $r$ (und $s$ ) relativ prim zu $\frac{P}{\delta}$ ist, so darf 
man die Congruenz (1) durch $\prod(r)=\prod^{(s)}$ dividieren, so dass man hat:

$$
\prod(\varepsilon) \equiv A^{\frac{1}{2} \varphi\left(\frac{P}{\delta}\right)}\left(\bmod \frac{P}{\delta}\right)
$$

Nach dem früher bewiesenen Hilfssatze ist aber

$$
\left.\begin{array}{l}
\operatorname{entweder} A^{\frac{1}{2} \varphi\left(\frac{P}{\delta}\right)}=\left(\bmod \frac{A}{a}\right) \\
\text { oder } \quad A^{\frac{1}{2} \varphi\left(\frac{P}{\delta}\right)}=1
\end{array}\right\}\left(\bmod \frac{P}{\delta}\right)
$$

je nachdem $\frac{P}{\delta}$ den einzigen Primfactor $a$ oder mehrere verschiedene Primfactoren enthält und es ist demnach

$$
\begin{array}{ll}
\text { entweder } & \\
\text { oder } & \prod(\varepsilon)=\left(\begin{array}{l}
A \\
a
\end{array}\right) \mid \\
&
\end{array}
$$

je nachdem der erste oder zweite dieser beiden Fälle eintritt.

Will man nun das Product aller möglichen $\varepsilon$-Größen, d. i. den Wert von $\varepsilon_{1} \varepsilon_{2} \ldots \frac{\varepsilon_{P-1}}{2}$ bestimmen, so muss man nach Früheren für $\delta$ der Reihe nach alle Factoren von $P$, mit Ausnahme von $P$ selbst, setzen, und die entsprechenden Werte für $\prod(\varepsilon)$ mit einander multiplicieren. Dabei wird auch $\frac{P}{\delta}$ die Reihe der Factoren von $P$ durchlaufen ( $P$ mit eingerechnet und nur die Einheit ausgenommen), da aber das entsprechende $\prod(\varepsilon)$ immer $=+1$ ist, so oft $\frac{P}{\delta}$ aus zwei oder mehreren verschiedenen Primzahlen zusammengesetzt ist, so hat man nur alle jene Werte von $\frac{P}{\delta}$ zu berücksichtigen, welche die Form einer Primzahl-Potenz besitzen; ist also $P=a^{\alpha} b^{\beta} c^{\gamma} .$. , so hat man successive $\mathrm{zu}$ setzen:

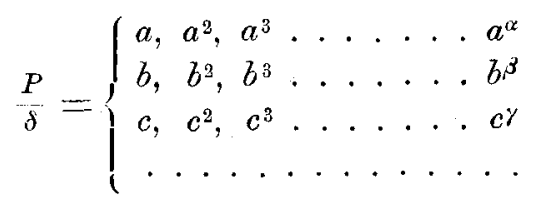

und erhält für jedes Element der ersten Horizontalreihe als: entsprechendes $\Gamma(\varepsilon)$ den Wert $\left(\frac{A}{a}\right)$, für jedes Glied der zweiten 
Horizontalreihe $\left(\frac{A}{b}\right)$ u. s. w. Da alle so erhaltenen Werte mit einander zu multiplicieren sind, so ergibt sich

$$
\begin{aligned}
\varepsilon_{1} \varepsilon_{2} \ldots \varepsilon_{\frac{\varepsilon_{-1}}{2}} & =\left(\frac{A}{a}\right)^{\alpha} \cdot\left(\frac{A}{b}\right)^{\beta} \cdot\left(\frac{A}{c}\right)^{\beta} \cdot \ldots \\
& =\left(\frac{A}{a^{\alpha}}\right) \cdot\left(\frac{A}{b^{\beta}}\right),\left(\frac{A}{c^{\gamma}}\right) \cdot \ldots \\
\text { oder } \varepsilon_{1} \varepsilon_{2} \ldots \varepsilon_{\frac{P-1}{2}} & =\left(\begin{array}{c}
A \\
P
\end{array}\right) \cdot \text { q. e. d. }
\end{aligned}
$$

Die Methode des vorangehenden Beweises, und speciell der Gleichungen (4), geben auch Anfschluss über die Vertheilung der positiven und negativen Vorzeichen in der Reihe $(B)$, was an einem Beispiel erprobt werden mag.

Es sei $P=45=3^{2} .5, A=8$, und wenn man der besseren Übersicht wegen unter jedes Glied der Reihe $(A)$ das correspondierende Glied von $(B)$ setzt; so hat man folgende Zusammenstellung:

$1 \cdot 8, \quad 2 \cdot 8, \quad 3 \cdot 8, \quad 4 \cdot 8, \quad 5 \cdot 8, \quad 6 \cdot 8, \quad 7 \cdot 8, \quad 8 \cdot 8, \quad 9 \cdot 8$, $+8,+16,-21,-13,-5,+3,+11,+19,-18$,

$10 \cdot 8,11 \cdot 8,12 \cdot 8,13 \cdot 8,14 \cdot 8,15 \cdot 8,16 \cdot 8,17 \cdot 8,18 \cdot 8,19 \cdot 8$, $-10,-2,+6,+14,+22,-15,-7,+1,+9,+17$,

$$
\begin{array}{r}
20 \cdot 8, \quad 21 \cdot 8, \quad 22 \cdot 8 . \\
-20,-12,-4 .
\end{array}
$$

Die Zahlen von 1 bis 22 zerfallen nun in 5 Gruppen, je nachdem sie zu 45 relativ prim sind, oder mit dieser Zahl den größten gemeinschaftlichen Theiler $3,3^{2}, 5,3.5$ besitzen. Diese Gruppen, sowie die ihnen entsprechende Vertheilung der Vorzeichen in der Reihe der absolut kleinsten Reste finden sich in der nebenstehenden Tabelle zusammengestellt. Man ersieht aus' derselben, dass die Anzahl der negativen Reste in der ersten und zweiten Gruppe eine gerade, in der dritten, vierten und fünften Gruppe dagegen eine ungerade ist, weil $\Gamma(\varepsilon)$ in den ersteren $=+1$, in letzteren aber $=-1$ ist, ein Verhalten, welches an der zweiten, mit $(B)$ bezeichneten Zeile der Tabelle direct beobachtet werden kann. Ebenso bestätigt sich die früher erwähnte Thatsache, dass die in einer solchen Gruppe von $(B)$ enthaltenen Reste, vom Vorzeichen und der Reihenfolge ab- 
472 Max Mand l: Üb. d. Verallgemeinerung eines Gauß'schen Algorithmus. gesehen, mit den Coefficienten $m$ derselben Gruppe übereinstimmen.

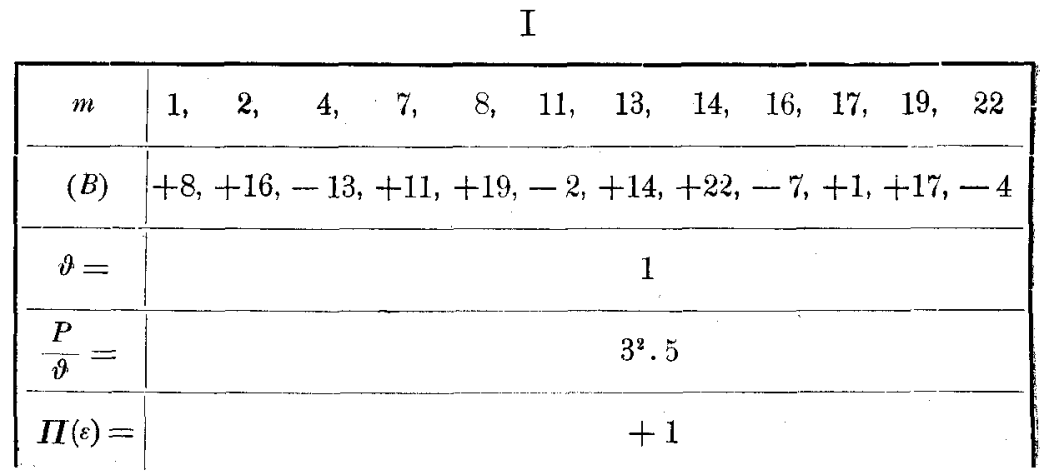

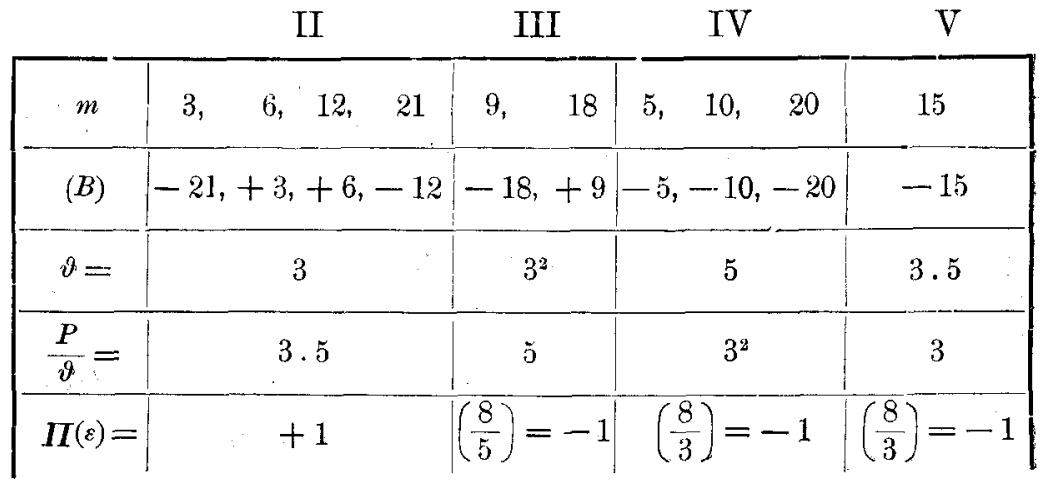

